International Conference on New Interfaces for Musical Expression

\title{
Camera Ready- RETURN
}

\section{BAI XIAOMO}

License: Creative Commons Attribution 4.0 International License (CC-BY 4.0). 


\section{RETURN (Sound Installation, Laser Installation)}

\section{BAI XIAOMO}

\section{PubPub Link}

https://nime.pubpub.org/pub/4wdlh1sh/draft?access=yez66tk3

\section{Conference Abstraction}

This project demonstrates a method of creating sound and laser devices using Max/MSP programming. The sound signal is also the control signal for driving the laser. Using the DMX protocol, the brightness of the red laser beam is controlled in real time and the intensity of the sound signal changes synchronously. This explains the characteristics of interactive sound devices: computer programming is used as a means, information is transformed into essence, cross-media interaction is the style, and sound is the core element of the work.

\section{Requirements (optional, especially for the performance on-site)}

- equipment: Laser Light, Black walnut, Acrylic, Loudspeaker, Computer, Mixer

- space: $180 \mathrm{~cm} \times 35 \mathrm{~cm} \times 38 \mathrm{~cm}$

\section{Program Description}

The sound and laser installation "RETURN" uses acrylic, lasers, and black walnut as materials. A 1.8-meter-long, 0.35-meter-wide, and 0.38-meter-high physics table is built on the nearly 200-year-old table in the center of the exhibition hall. The matrix is equidistantly distributed with nine acrylic plates, they are parallel to each other, and each acrylic plate is printed with a pattern generated by a computer program. A red laser beam pierced nine layers of acrylic from south to north. The sound part of the work is composed of five paragraphs, all of which are generated in real time at the exhibition site using the Max/MSP program. The sound signal is also a control signal for driving the laser. The brightness of the red light beam changes simultaneously with the strength of the sound signal. This work is also a continuation of the characteristics of Bai Xiaomo's "Order" series of interactive installations: computer programming is used as a means, information is transformed into essence, cross-media interaction is the style, and sound is the core element of the work. 


\section{Media}

https://vimeo.com/516206117

or

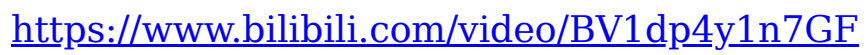

\section{ACKNOWLEDGEMENTS}

This project is supported by the Sichuan Provincial Key Laboratory of Digital Media Art. 